\title{
Utilização da cirurgia de mohs no tratamento de neoplasias malignas não melanocíticas da pele - uma revisão sistemática
}

João Augusto Gomes de Souza Monteiro de Brito ${ }^{1}$ (D), Lucas da Silva Lopes ${ }^{1}$ (D) Ana Luísa Gross

Rodrigues $^{1}$ (i), Caio Vinicius Botelho Brito ${ }^{1}$ (D)

\section{RESUMO}

Objetivo: Averiguar a efetividade da utilização da cirurgia de MOHS no manejo de tumores cutâneos tipo não melanoma em comparação a outros métodos de terapia. Métodos: O estudo consiste em uma revisão sistemática, cuja coleta de artigos ocorreu nas bases de dados MEDLINE, PubMed Central (PMC), LILACS e SciELO, utilizando os termos "Cirurgia de MOHS", "Câncer de pele", "Ensaio clínico", excluindo "melanoma". Resultados: Do total de 132 artigos identificados, foram considerados elegíveis 11. A análise dos artigos demonstrou que a CMM, comparada a outros métodos, apresentou resultados estéticos melhores, além de menor grau de complicações em tumores recorrentes e um custo/benefício variável com o país onde foi feita operação. A CMM também apresentou menores índices de recorrência em todos os estudos. Conclusão: No geral, a cirurgia de MOHS se mostrou um método terapêutico promissor. No entanto, existem ainda poucos estudos comparativos sobre a eficácia da CMM e os que existem se concentram em poucas regiões do mundo.

Palavras-chave: Cirurgia de MOHS; Câncer de pele; Revisão sistemática.

1. Universidade do Estado do Pará. Curso de Medicina. Belém (PA), Brasil. 


\section{INTRODUÇÃO}

A cirurgia micrográfica de Mohs (CMM) é um procedimento metódico e inovador, conhecido por ser uma das formas mais eficazes de tratar diversas neoplasias malignas da pele, por oferecer maiores taxas de cura e maior conservação de tecido saudável em comparação com outros métodos. Trata-se de uma técnica desenvolvida pelo Dr. Frederic Mohs na década de 1930 com o princípio orientador de excisões microscopicamente controladas. ${ }^{1}$

Nela, o tumor é extraído, mapeado com precisão e processado com fragmentos horizontais que são congelados para avaliação histológica intraoperatória. O processo é repetido com o tumor removido cirurgicamente, camada por camada, e cada camada é examinada microscopicamente. A remoção continua até que não permaneçam células anormais, permitindo a remoção completa do tumor e conservação máxima de tecido não comprometido. A avaliação imediata da margem leva às maiores taxas de cura e seu uso é muitas vezes parte de uma abordagem multidisciplinar para o tratamento de tumores cutâneos. ${ }^{2,3}$

Nesse contexto, cabe ressaltar que o tratamento cirúrgico é a base da terapia de muitos tumores de pele. Os carcinomas basocelulares (CBC) compreendem a maioria desses e, em geral, apresentam um curso clínico lento e indolente, com baixo risco de metástase. No entanto, podem ser localmente invasivos, causando extensa destruição tecidual. Nesse sentido, a cirurgia micrográfica de Mohs, é o tratamento de escolha para os tumores de alto risco, em locais onde a conservação de tecidos é de extrema importância ${ }^{4}$, além de estudos relatarem uma menor taxa de recorrência quando comparada com a excisão cirúrgica convencional. ${ }^{5}$

Assim como para os carcinomas basocelulares, estudos demonstraram que pacientes com outros tumores de pele não melanoma agressivos ou de alto risco também se beneficiam de métodos como MMS ou outras cirurgias controladas por margem, as quais diminuem as taxas de recorrência, apresentam altos índices de cura e melhores resultados cosméticos, com maior preservação de tecido, recomendando seu uso no tratamento de lesões, especialmente, da zona $\mathrm{H}$ da face e nos tumores de subtipo histológico agressivo. $6,7,8$

O estudo de Reeder et al. (2015) ${ }^{9}$ observou um aumento na utilização da cirurgia de Mohs no tratamento de neoplasias de pele entre 1995 a 2010.
Apesar disso, a taxa global de utilização desse procedimento permanece baixa quando comparada ao método convencional.

Além dos métodos cirúrgicos de tratamento, terapias direcionadas menos invasivas estão sob estudo ativo e podem, eventualmente, substituir algumas terapias cirúrgicas convencionais em um futuro próximo, diversificando as formas de manejo de tumores de pele não melanoma. ${ }^{10}$

Nesse sentido, a presente pesquisa visa fornecer à comunidade científica e aos profissionais de saúde conhecimento acerca das vantagens e importância da utilização da técnica cirúrgica micrográfica de Mohs no tratamento de tumores cutâneos não melanocíticos, com base nas evidências mais confiáveis disponíveis na literatura médica sobre o assunto. Assim, por meio de revisão sistemática, este trabalho tem como objetivo sintetizar e averiguar a efetividade da utilização da cirurgia micrográfica de Mohs em tumores cutâneos não melanocíticos em comparação com outros métodos de tratamento.

\section{MÉTODOS}

Trata-se de uma revisão de literatura do tipo sistemática na qual foi realizada uma pesquisa nas bases de dados eletrônicas seguindo as recomendações da Preferred Reporting Items for Systematic Reviews e Meta-Analyses (PRISMA) ${ }^{11}$.

A coleta de artigos foi realizada por busca nas bases de dados eletrônicas MEDLINE, PubMed Central (PMC), LILACS E SCIELO.

Os descritores utilizados foram "Cirurgia de MOHS", "Câncer de pele" e "Ensaio clínico" e seus sinônimos em português, inglês e espanhol, excluindo "melanoma" e seus sinônimos.

O processo de busca foi realizado de forma independente e isolado por cada um dos autores. Primeiramente, foi levantada a totalidade de artigos identificados nas bases de dados pelos sistemas de busca PUBMED, Biblioteca Virtual em Saúde (BVS) e o próprio da base SciELO e organizados em tabelas no Microsoft Excel 2016 com posterior eliminação de artigos duplicados. Os títulos e resumos dos artigos encontrados foram então lidos para verificação da elegibilidade para o presente estudo. Após exclusão de artigos que não foram compatíveis, foi realizada a leitura na íntegra para mais uma verificação da elegibilidade. Posteriormente, as informações encontradas foram cruzadas entre os autores e 
verificadas as diferenças nos resultados obtidos, de modo a reduzir possíveis falhas nas buscas em bases de dados. Por fim, os dados foram sintetizados de forma primariamente qualitativa.

Foram selecionados os artigos de estudos primários que compararam a cirurgia micrográfica de Mohs com outras formas de tratamento como forma terapêutica para tumores de pele não melanoma, com resumos disponíveis nos bancos de dados informatizados selecionados e texto disponível na íntegra na internet ou fornecido pela fonte original, e considerados artigos publicados de 1995 até 2019.

Foram excluídos artigos que estudavam melanoma, que não possuíam metodologia ou resultados claros, que não realizaram método de comparação entre terapêuticas e que seus dados completos não foram encontrados.

Foi realizada uma ampla revisão qualitativa dos estudos selecionados, de onde foram extraídas informações sobre a efetividade dos métodos de tratamento, grau de recorrência de tumores após cirurgia, resultado estético e de qualidade de vida, custo/benefício e complicações. Após isso, foi realizada a elaboração de gráficos e tabelas para a exposição dos resultados.

Conforme demonstra a Figura 1, foram encontradas 132 potenciais citações. Após a primeira leitura, onde foram excluídos os estudos duplicados (23), 90 foram removidos durante a avaliação de títulos e resumos. Dentre os 19 selecionados, oito artigos foram excluídos durante a aplicação dos critérios de inclusão e exclusão.

Dos 11 artigos incluídos, dez são originários da base de dados MEDLINE e um da PMC. Artigos das demais bases de dados não foram elegíveis para o estudo.

\section{RESULTADOS}

A Tabela 1 sintetiza características dos artigos originais que compõem a amostra e a Tabela 2 descreve procedimentos metodológicos e principais resultados.

O estudo incluiu 11 artigos, dos quais a maioria são estudos caso-controle e ensaio clínico randomizado. No total, foram incluídos na revisão dados de 3.095 pacientes. Os trabalhos que compuseram o estudo foram oriundos dos países: Estados Unidos da América (EUA), Reino Unido e Holanda. Nem um trabalho das bases de dados LILACS e SciELO foi elegível para o estudo.
Figura 1 Fluxograma de seleção dos artigos.
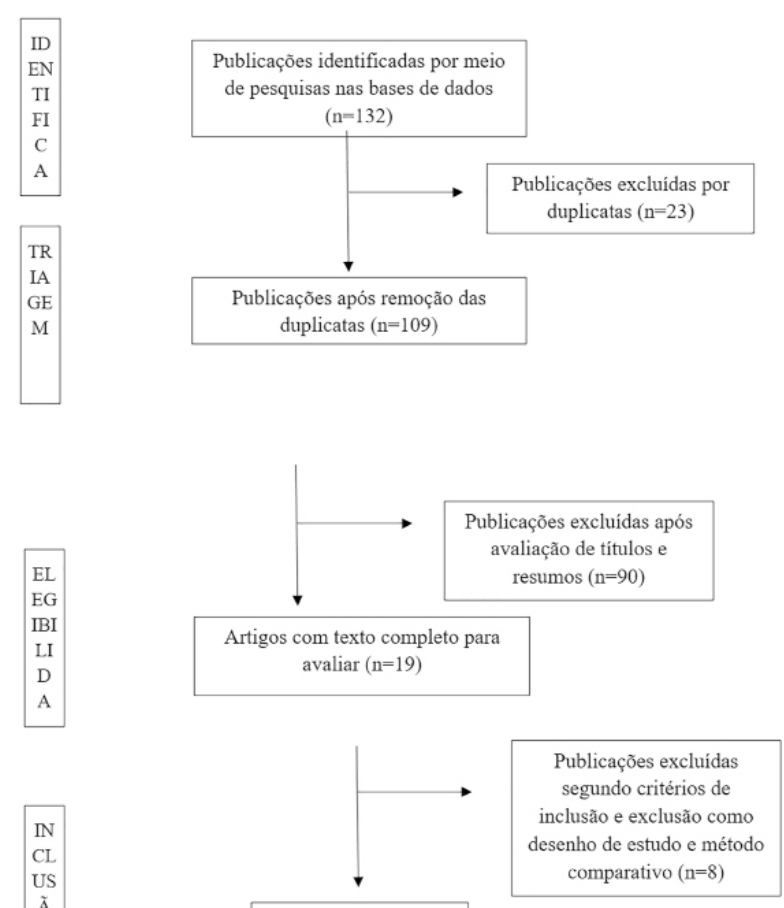

Artigos incluidos

Adaptado de PRISMA (Preferred Reporting Items for Systematic Reviews and Meta-analysis; http://www.prisma-statement.org/ index.html).

O estudo teve uma porcentagem maior de pacientes que apresentaram lesões na cabeça e pescoço e do tipo histológico Carcinoma Basocelular. Nove dos onze artigos buscaram comparar a excisão cirúrgica convencional com o método micrográfico de Mohs.

\section{DISCUSSÃO}

Todos os artigos avaliados apresentaram menores índices de recorrência de carcinoma basocelular após tratamento com cirurgia micrográfica de Mohs, em comparação com outros métodos ${ }^{12-14}$. No entanto, nem um deles apresentou significância estatística, o que pode ter ocorrido pelo fato de nem um dos estudos ter feito um acompanhamento de no mínimo cinco anos para avaliação, como foi apontado por Smeets e colaboradores (2004) ${ }^{14}$.

O estudo de Davis e colaboradores (1997) ${ }^{15}$ evidenciou que o tratamento de fibroxantoma atípico com CMM resultou em uma taxa de recorrência e metástase menor do que com ampla excisão, além de maior conservação de tecido normal. Resultado 
semelhante ao encontrado em metanálise feita com 23 estudos com 907 pacientes, com taxa de recorrência após cirurgia de Mohs e excisão local ampla, respectivamente, de $2 \%$ e $8,7 \%$. O controle cuidadoso e completo da margem através do CMM provavelmente contribui para os melhores resultados, em particular nos pacientes imunocomprometidos que parecem ter maior risco de recorrência. ${ }^{16}$
Estudo de Waine et al. (2015) ${ }^{19}$, que avaliou as técnicas reconstrutivas usadas após cirurgia de Mohs em comparação a resultados que seriam obtidos com excisão convencional (ECC), evidenciou que, se a ECC tivesse sido utilizada, $56 \%$ dos pacientes teriam recebido uma reconstrução mais invasiva ou esteticamente menos desejável e $24 \%$ das margens permaneceram positivas. Como tal, $80 \%$

Tabela 1 Descrição dos estudos incluídos.

\begin{tabular}{|c|c|c|c|c|}
\hline Citação & Tipo de estudo & Local & Ano & Amostra \\
\hline Davis et al. & Caso-controle & EUA & 1997 & 116 pacientes \\
\hline Boyer et al. & Caso-controle & EUA & 2002 & 45 pacientes \\
\hline Dubay et al. & Caso-controle & EUA & 2004 & 62 pacientes \\
\hline Essers et al. & $\begin{array}{l}\text { Ensaio clínico } \\
\text { randomizado prospectivo }\end{array}$ & Holanda & 2006 & 565 pacientes \\
\hline Wain et al. & Caso-controle & Reino Unido & 2015 & 157 pacientes \\
\hline Patel et al. & $\begin{array}{l}\text { Coorte prospectiva com } \\
\text { fase retrospectiva }\end{array}$ & EUA & 2017 & 369 pacientes \\
\hline Muller et al. & $\begin{array}{l}\text { Ensaio clínico } \\
\text { randomizado duplo cego }\end{array}$ & Reino Unido & 2009 & 30 pacientes \\
\hline Chren et al. & $\begin{array}{l}\text { Estudo de coorte } \\
\text { prospectivo }\end{array}$ & EUA & 2007 & 633 pacientes \\
\hline Smeets et al. & $\begin{array}{l}\text { Ensaio clínico } \\
\text { randomizado }\end{array}$ & Holanda & 2004 & $\begin{array}{l}374 \text { pacientes com } 408 \text { neoplasias } \\
\text { primárias }\end{array}$ \\
\hline Cook et al. & Caso-controle & EUA & 1998 & 400 tumores \\
\hline Ravitskiy et al. & Caso-controle & EUA & 2012 & 344 pacientes e 406 tumores \\
\hline
\end{tabular}

Fonte: próprio autor

Para o tratamento de dermatofibrossarcoma protuberante, o estudo de DuBay et al. (2004) $)^{17}$ demonstrou que tanto a excisão local ampla como a cirurgia de Mohs e a abordagem combinada parecem ser eficazes e com altas taxas de controle local. Ao contrário de outro estudo no qual se verificou que a recorrência do DFSP após excisão ampla e CMM era $9,10 \%$ e 2,72\%, respectivamente, após um tempo médio de acompanhamento de 5,32 anos. Tal resultado pode ter ocorrido devido ao fato de o serviço de cirurgia do estudo de DuBay utilizar um método diferente do padrão mostrado na literatura, com ampla excisão local em forma de diamante e com avaliação completa da margem periférica.

O estudo de Boyer et al. (2002) ${ }^{18}$ também indicou baixa taxa de recorrência (cerca de 4\%) após excisão de carcinoma de células de Merkel por método de Mohs, sem métodos complementares de controle das lesões como a radiação adjuvante. dos pacientes submetidos à CMM e subsequente reconstrução tiveram um resultado melhor. Resultado análogo ao de Smeets et al. (2004) ${ }^{14}$ e de Dubay $(2004)^{17}$ que evidenciaram um tamanho médio do defeito após ECC maior, em comparação a CMM para tumores recorrentes e mais agressivos. No entanto, não houve diferença estatística importante em relação aos carcinomas primários. Nesse sentido, a CMM seria preferível para tumores agressivos para evitar defeitos maiores, mau resultado estético e problemas funcionais.

A despeito da avaliação da qualidade de vida nos pacientes, Essers et al. (2006) ${ }^{13}$ demonstrou não haver diferença estatística significante no nível de ansiedade e boa qualidade de vida após seis meses da realização da cirurgia em comparação com a linha de base entre pacientes submetidos a CMM ou excisão cirúrgica. Situação semelhante à encontrada em outro estudo ${ }^{20}$, o qual identificou que pacientes tratados com excisão ou cirurgia de Mohs tiveram 
Tabela 2 Descrição dos aspectos metodológicos e principais desfechos dos estudos incluídos nesta revisão.

\begin{tabular}{llll}
\hline Citação Intervenção & Tipo histológico & $\begin{array}{l}\text { Local da } \\
\text { lesão }\end{array}$ & Principais resultados/conclusões
\end{tabular}

\begin{tabular}{|c|c|c|c|c|}
\hline $\begin{array}{l}\text { Davis } \\
\text { et al. }\end{array}$ & CMM e ECC & Fibroxantoma atípico & $\begin{array}{l}78 \% \text { em } \\
\text { cabeça e } \\
\text { pescoço }\end{array}$ & $\begin{array}{l}\text { Não houve recidivas em pacientes com MMS } \\
\text { em } 26,6 \text { meses. } \\
3 \text { pacientes submetidos a ECC } \\
\text { apresentaram recorrência. } \\
\text { O controle microscópico das margens } \\
\text { cirúrgicas com MMS resulta em uma taxa } \\
\text { de recorrência e metástase menor do que } \\
\text { com ECC e conserva o tecido normal. }\end{array}$ \\
\hline $\begin{array}{l}\text { Boyer } \\
\text { et al. }\end{array}$ & $\begin{array}{l}\text { CMM apenas e } \\
\text { radiação + CMM }\end{array}$ & $\begin{array}{l}\text { Carcinoma de células } \\
\text { de Merkel }\end{array}$ & $\begin{array}{l}\text { Cabeça e } \\
\text { pescoço mais } \\
\text { comuns }\end{array}$ & $\begin{array}{l}\text { No grupo submetido apenas CMM } \\
\text { apresentou } 1 \text { recorrência marginal e } 3 \\
\text { metástases. } \\
\text { No grupo submetido às } 2 \text { intervenções, } 0 \\
\text { recorrência ou metástase. } \\
\text { Os tratamentos não diferiram } \\
\text { significativamente para a sobrevida global e } \\
\text { específica dos pacientes } \\
\text { A taxa de recorrência de } 4 \% \text { valida } \\
\text { a cirurgia de Mohs sozinha como um } \\
\text { tratamento eficaz. }\end{array}$ \\
\hline $\begin{array}{l}\text { Dubay } \\
\text { et al. }\end{array}$ & $\begin{array}{l}\text { ECC, CMM e } \\
\text { abordagem } \\
\text { combinada: oncologia } \\
\text { cirúrgica, CMM, } \\
\text { cirurgia plástica, } \\
\text { cirurgia plástica } \\
\text { otorrinolaringológica- } \\
\text { facial, oftalmologia, } \\
\text { cirurgia orto-maxilo- } \\
\text { facial e oncologia por } \\
\text { radiação. }\end{array}$ & $\begin{array}{l}\text { Dermatofibrossarcoma } \\
\text { protuberante }\end{array}$ & $\begin{array}{l}\text { Tronco e } \\
\text { extremidades } \\
\text { (48) e face } \\
(15)\end{array}$ & $\begin{array}{l}\text { As lesões tratadas pela CMM menores em } \\
\text { comparação às tratadas com abordagem } \\
\text { multidisciplinar ou grande excisão } \\
\text { O defeito pós-operatório da CMM foi menor } \\
\text { comparado aos defeitos após abordagem } \\
\text { combinada multidisciplinar ou excisão ampla. } \\
\text { Todas as modalidades de tratamento, } \\
\text { selecionadas por fatores do tumor e do } \\
\text { paciente foram capazes de alcançar taxas de } \\
\text { controle local muito altas. }\end{array}$ \\
\hline
\end{tabular}

Grupo primário: 5 recorrências após ECC e 3 após CMM em 30 meses.

Grupo recorrente: 3 recidivas após excisão cirúrgica e para CMM nenhuma.

Essers ECC e CMM CBC

Cabeça em Os custos da CMM foram mais altos.

et al. ECC e CMM CBC todos os As curvas de aceitabilidade para o CBC primário e recorrente demonstraram que a probabilidade de CMM ser mais efetiva em termos de custo e excisão cirúrgica não chegou a $50 \%$ e a relação de custo-benefício ainda é alta demais para recomendar uma ampla implementação.

ECC: $24 \%$ teriam tido margem histológica incompleta e $9 \%$ teriam perdido estruturas fundamentais de forma desnecessária. $80 \%$ dos submetidos a CMM tiveram resultados melhores comparados a ECC Critérios de referência detalhados, pré-

Wain
et al. Região nasal operatório completo, avaliação do paciente, em $48 \%$ dos apreciação das habilidades e limites da ECC casos permitiram ao serviço produzir um benefício cirúrgico demonstrável em pelo menos $80 \%$ dos pacientes submetidos à CMM quando comparados ao ECC.

A comparação de CMM e ECC fornece um método para avaliar a eficiência e efetividade do serviço de CMM 
Patel
et al. CMM e BE $\quad \mathrm{CBC}$ e CEC

Muller CMM e ECC CBC Cabeça e

et al.

pescoço (12)

ECC, CMM e

Chren eletrodissecação + curetagem.

Smeets

et al.

CMM e ECC

$\mathrm{CBC}$

Todas na cabeça
$99,5 \%$ da BE e $100,0 \%$ das lesões tratadas com CMM estavam livres de recorrência. Resultados estéticos: "excelente" ou "bom" em $97,6 \%$ das lesões tratadas com BE e $95,7 \%$ das lesões tratadas com CMM. BE é uma opção não cirúrgica efetiva de tratamento para câncer de pele não melanoma em grau inicial.

A área média dos defeitos cirúrgicos no grupo CMM foi de $116,6 \mathrm{~mm} 2$, contra 187,7 $\mathrm{mm} 2$ no grupo de ECC, de modo que a área acometida pela cirurgia padrão é 1,6 vezes maior do que com CMM.

Os tratados com ECC ou CMM apresentaram melhorias estatisticamente significativas nos três domínios da qualidade de vida Os pacientes tratados com eletrodissecação e curetagem não apresentaram alterações na qualidade de vida relacionada ao tumor Os escores médios dos sintomas de Skindex melhoraram 9,7 (IC 95\%: 6,9, 12,5)

após a excisão, 10,2 (7,4, 12,9) após a cirurgia de Mohs e 3,4 ( $\pm 0,9,7,6)$ após eletrodissecação + curetagem

Carcinomas primários: 3\% recorreram após o ECC e $2 \%$ após CMM.

Carcinomas recorrentes: 3\% de recorrência após ECC e 0 após CMM.

Os custos operacionais totais do CMM foram mais altos do que os de ECC.

Nenhuma conclusão definitiva sobre as taxas de recorrência de CBC primários ou recorrentes ainda é possível.

CMM $x$ métodos tradicionais de excisão: excisão

Cook com margem de

et al. seção permanente,

CBC e CCE

Cabeça e pescoço excisão com controle de margem de seção congelada

Ravitskiy CMM x ECC com et al.
(346)

Extremidades e genitália externa (22)

Cabeça

(275)

Tronco e extremidade (79)
Mohs é um método de excisão cirúrgica com alto valor sistêmico econômico em comparação à excisão cirúrgica tradicional

CMM foi a mais barata por tumor. Este estudo confirma a CMM como a pedra angular do tratamento econômico, independentemente do local de serviço ou tipo de patologia.

Fonte: próprio autor

CMM: Cirurgia Micrográfica de Mohs; ECC: Excisão Cirúrgica Convencional; BE: Braquiterapia Eletrônica; CBC = Carcinoma Basocelular; $\mathrm{CEC}=$ Carcinoma Espinocelular.

resultados de qualidade de vida similares, porém, melhores que os obtidos após eletrodissecação e curetagem. No entanto, é possível que instrumentos genéricos como o NHP e o STAI, utilizados na pesquisa de Essers ${ }^{13}$ para avaliar tais questões, não sejam sensíveis o suficiente para detectar efeitos e diferenças dos tratamentos na vida dos pacientes.
Quanto ao grau de tecido poupado, o estudo de Muller e colaboradores (2009) ${ }^{21}$ verificou que a área de tecido retirada na excisão cirúrgica convencional é 1,6 vezes maior do que após a cirurgia micrográfica de Mohs, algo que entra em conflito com o que foi encontrado por Smeets et al. (2004) ${ }^{14}$, onde não foram observadas diferenças significativas. No entanto, tal fato pode ser explicado pelo fato de 
que no trabalho de Smeets e colaboradores foram utilizadas margens de $3 \mathrm{~mm}$ em ambos métodos terapêuticos, sendo que, usualmente, a CMM utiliza margens bem menores, algo apontado nos dois artigos, enquanto no trabalho de Muller et al. ${ }^{21}$ foram adotadas margens de $2 \mathrm{~mm}$ para CMM e $4 \mathrm{~mm}$ para ECC. Ainda assim, nos casos em que eram necessárias mais de uma ECC ou mais de um estágio de CMM, a CMM demonstrou poupar muito mais tecido.

No que diz respeito ao custo isolado da CMM, é um consenso que ele é maior do que o da excisão cirúrgica convencional (ECC), principalmente, pelo fato da CMM exigir um maior tempo de cirurgia e pelos custos com análise histopatológica. ${ }^{13,14,22}$ No entanto, ao considerar o custo/benefício em diferentes situações, ocorre divergência. Essers e colaboradores (2006) ${ }^{13}$ verificaram que em nenhuma das situações a CMM possui um custo/benefício melhor tanto em $\mathrm{CBC}$ primário quanto recorrente para implementação em larga escala, mas que maiores estudos poderiam eventualmente evidenciar melhor custo/benefício para casos recorrentes e para localizações específicas como em orelhas. Cook et al. (1998) ${ }^{23}$ e Ravitsky e colaboradores (2012) ${ }^{22}$, por outro lado, verificaram que, mesmo com alto custo, a CMM possui um melhor custo/benefício que métodos tradicionais de cirurgia. Uma possibilidade para a diferença significativa entre os resultados talvez decorra da realidade em que os estudos foram inseridos, pois o estudo de Essers ocorreu na Holanda, enquanto o de $\operatorname{Cook}^{23}$ e o de Ravitsky ${ }^{22}$ ocorreram nos Estados Unidos da América.

Os resultados acerca de complicações ocorreram de forma similar entre os estudos. Comparando CMM com ECC, os estudos de Smeets e colaboradores (2004) $)^{14}$ e Essers e colaboradores (2006) ${ }^{13}$ demonstraram não haver diferença significativa nas taxas de complicações para $\mathrm{CBC}$ primário. No entanto, para o $\mathrm{CBC}$ recorrente, os dois trabalhos encontraram uma taxa de $19 \%$ para ECC e 8\% para CMM, evidenciando superioridade da cirurgia micrográfica de MOHS para essas situações. Comparando com a braquiterapia eletrônica (BE), apesar da CMM ter apresentado menor índice de complicações em quase todos os tipos de complicações avaliados, os dois métodos apresentaram resultados semelhantes com baixos índices, exceto no que diz respeito a telangiectasias, que foram muito mais comuns em BE $(31,4 \%)$ do que após CMM $(11,1 \%)$.

\section{CONCLUSÃO}

A cirurgia micrográfica de Mohs é um método terapêutico de comprovada eficácia para câncer de pele não melanoma com baixos índices de recorrência, ótimos resultados estéticos e baixas taxas de complicações. Comparando com outros métodos, a CMM apresentou resultados estéticos comprovadamente melhores que a ECC, além de menor grau de complicações em tumores recorrentes e um custo/benefício variável com o local do estudo. Com relação ao grau de recorrência, a CMM apresentou menores índices em todos os estudos, apesar de alguns não apresentarem significância estatística. Em um panorama geral, ainda são escassos estudos comparativos sobre a eficácia da CMM e os que existem se concentram em poucas regiões do mundo. Com isso, maiores estudos são necessários para confirmar a cirurgia micrográfica de Mohs como um método, de fato, superior à excisão cirúrgica convencional para o tratamento de câncer de pele não melanoma.

\section{REFERÊNCIAS}

1. Ladda MA, Lynde CW. Mohs Micrographic Surgery: Development of the Technique. J cutan medic surger. $2019 ; 23(2): 236$.

2. Wong E, Axibal E, Brown M. Mohs Micrographic Surgery. Facial Plast Surg Clin North Am. 2019; 27(1): 15-34.

3. Van loo E, Mosterd K, Krekels GA, Roozeboom $\mathrm{MH}$, Ostertag JU, Dirksen CD, et al. Surgical excision versus Mohs' micrographic surgery for basal cell carcinoma of the face: a randomised clinical trial with 10 year followup. Eur J Cancer. 2014; 50(17): 3011-20.

4. Kauvar AN, Cronin JRT, Roenigk R, Hruza G, Bennett R. Consensus for nonmelanoma skin cancer treatment: basal cell carcinoma, including a cost analysis of treatment methods. Dermatol Surg. 2015; 41(5): 550-71.

5. Tanese K. Diagnosis and Management of Basal Cell Carcinoma. Curr Opin Oncol. 2019; 20(2): 1-13.

6. Murray C, Silva JD, Hanna TP, Bradshaw S, Solish N, Moran B, et al. Patient indications for Mohs micrographic surgery: a clinical practice guideline. Curr Opin Oncol. 2019; 26(1): 94-99.

7. Sanchez FH, Estefan JL, Estefan LL. Cirurgia micrográfica de Mohs: estudo de 93 tumores operados em um centro de referência no Rio de Janeiro. Surg Cosm Derm. 2013; 5(3): 206-10. 
8. Pontes LT. Cirurgia micrográfica de Mohs em um serviço universitário: estudo retrospetivo de 5 anos [dissertação]. Campinas: Universidade Estadual de Campinas, Faculdade de Ciências Médicas; 2016.

9. Reeder VJ, Gustafson C J, Mireku K, Davis SA, Feldman SR, Pearce DJ. Trends in Mohs surgery from 1995 to 2010: an analysis of nationally representative data. Derm Surg. 2015; 41(3): 397-403

10. Correia STR, Silva R, Lopes JM. Carcinoma basocelular da pele (parte 2): diagnóstico, prognóstico e manejo. Future Oncology. 2015; 11(22): 3023-38.

11. Moher D, Liberati A, Tetzlaff J, Altman DG, PRISMA Group. Itens de relatório preferidos para revisões sistemáticas e metanálises: a declaração PRISMA. PLoS Med. 2009; 6(7): e1000097.

12. Patel R, Strimling R, Doggett $S$, Willoughby M, Miller $K$, Dardick $L$, et al. Comparison of electronic brachytherapy and Mohs micrographic surgery for the treatment of early-stage non-melanoma skin cancer: a matched pair cohort study. J Contemp Brachytherapy. 2017; 9(4): 338-44.

13. Essers BAB, Dirksen CD, Nieman FHM, Smeets NWJ, Krekels GAM, Prins MH, et al. Cost-effectiveness of Mohs Micrographic Surgery vs Surgical Excision for Basal Cell Carcinoma of the Face. Arch Dermatol. 2006; 142(2): 187-94.

14. Smeets NWJ, Krekels GAM, Ostertag JU, Essers BAB, Dirksen CD, Nieman FHM, et al. Surgical excision vs Mohs' micrographic surgery for basal-cell carcinoma of the face: randomised controlled trial. Lancet. 2004; 364(9447): 1766-72.

15. Davis JL, Randle HW, Zalla MJ, Roenigk RK, Brodland DG. A comparison of Mohs Micrografic Surgery and wide excision for the treatment of atypical fibroxantoma. Dermatol Surg. 1997; 23(2): 105-10.
16. Tolkachjov SN, Kelley BF, Alahdab F, Erwin PJ, Brewer JD. Atypical fibroxanthoma: Systematic review and meta-analysis of treatment with Mohs micrographic surgery or excision. J Am Acad Dermatol. 2008; 79(5): 929-34.

17. Dubay D, Cimmino V, Lowe L, Johnson TM, Sondak VK. Low Recurrence Rate after Surgery for Dermatofibrosarcoma Protuberans: a multidisciplinary approach from a single institution. Cancer. 2004; 100(5): 1008-16.

18. Boyer JD, Ziteli JA, Brodland DG, D Angelo G. Local control of primary Merkel cell carcinoma: Review of 45 cases treated with Mohs micrographic surgery with and without adjuvant radiation. J Am Acad Dermatol. 2002; 47(6): 885-92.

19. Wain RAJ, Tehrani H. Reconstructive outcomes of Mohs surgery compared with conventional excision: A 13-month prospective study. Journal of Plastic, Reconstructive \& Aesthetic Surgery. 2015; 68(7): 946-52

20. Chren MM, Sahay AP, Bertenthal DS, Sen S, Landerfeld CS. Quality of Life Outcomes of Treatments for Cutaneous Basal Cell Carcinoma and Squamous Cell Carcinoma. Journal of Investig Dermatol. 2007; 127(6): 1351-57.

21. Muller FM, Dawe RS, Moseley H, Fleming CJ. Randomized Comparison of Mohs Micrographic Surgery and Surgical Excision for Small Nodular Basal Cell Carcinoma: Tissue-Sparing Outcome. Dermatol Surg. 2009; 35(9): 1349-54.

22. Ravitskiy L, Brodland DG, Zitelli JA. Cost Analysis: Mohs Micrographic Surgery. Dermatol Surg. 2012; 38(4): 585-94.

23. Cook J, Ziteli JA. Mohs micrographic surgery: A cost analysis. J Am Acad Dermatol. 1998; 39(5): 698-703 
Autor Correspondente:

João Augusto Gomes de Souza Monteiro de Brito

joaoaugustobrito@hotmail.com

Editor:

Prof. Dr. Paulo Henrique Manso

Recebido: 01/06/2020

Aprovado: $14 / 04 / 2021$ 